\title{
Redes de ciudades en China imperial temprana (siglos III a.e.c.-IIl e.c.). Control territorial y lógicas de parentesco
}

\author{
Ignacio Villagrán \\ Universidad de Buenos Aires / Universidad Nacional de General Sarmiento, Argentina
}

\author{
Melina Fini \\ Universidad de Buenos Aires, Argentina
}

Fecha de recepción: 25 de julio de 2021. Fecha de aceptación: 18 de agosto de 2021.

\begin{abstract}
Resumen $^{1}$
El área de la llanura fértil del río Amarillo en el norte de China se caracteriza por la temprana aparición y acelerada proliferación de los núcleos urbanos, y el rápido desarrollo de las ciudades amuralladas desde el siglo XV a.e.c. En este artículo, nos proponemos investigar las lógicas de parentesco subyacentes en el entramado de ciudades durante el período imperial temprano, con especial énfasis en la dinastía Han (206 a.e.c.-220 e.c.). Revisaremos la fundación de ciudades en relación con los procesos de expansión territorial y de consolidación de un esquema de gobierno que mantuvo las tensiones entre los magistrados designados para cumplir con funciones administrativas en las provincias del imperio, por un lado, y los nobles hereditarios que formaban parte del clan imperial, por el otro. A pesar de los problemas que se derivaban de la duplicación de figuras de poder en los principales centros urbanos del
\end{abstract}

1 El presente artículo es una versión revisada de la charla "Ciudades y urbanismo en China antigua" ofrecida en el Ciclo de Conferencias "Ciudades y Urbanismo en el Mundo antiguo" organizado por el Instituto de Historia Antigua Oriental, Facultad de Filosofía y Letras, UBA, el 20 de agosto del 2020. Esta primera presentación fue ampliada con la contribución de Melina B. Fini para ser presentada en las "Jornadas Patagónicas de Estudios sobre Sociedades Antiguas" organizadas por la Universidad Nacional de la Patagonia Austral - Unidad Académica San Julián los días 27 y 28 de octubre de 2020. Agradecemos la invitación del Dr. Pablo Jaruf a participar del ciclo de charlas que sirvió de disparador del presente trabajo, y a la Dra. Magdalena Magneres y el Dr. Ianir Milevski por la invitación a la Mesa sobre Cercano y Lejano Oriente de las "Jornadas Patagónicas de Estudios sobre Sociedades Antiguas". 
imperio temprano, fue gracias a este esquema de gobierno dual que las redes de ciudades permitieron la expansión y control territorial que facilitó el gobierno de la dinastía Han por más de cuatro siglos.

Palabras clave: China antigua, ciudades, nobles hereditarios, parentesco, gobierno

City Networks in Early Imperial China (III BCE-III CE). Territorial Control and Kinship Logics

\begin{abstract}
The fertile area of the Yellow River valley in the north of China is characterized by the early rise of urban settlements, and the rapid development of walled cities since at least the 15th century BCE. In this article we propose to study the kinship logics that underlaid the network of cities during the early imperial period, with a special emphasis on the Han dynasty (206 BCE-220 CE). We will revisit the foundation of cities in relation with the processes of territorial expansion and the consolidation of a government structure that maintained the tensions between magistrates designated by the central court to fulfill administrative duties in the provinces of the empire, on the one hand; and the hereditary nobles that were part of the imperial clan, on the other. In spite of the problems of the duplication of figures of authority in the main urban centers of the empire, it was in fact due to this dual-government structure that the networks of cities allowed for the territorial expansion and control that sustained the four centuries rule of the Han dynasty.
\end{abstract}

Keywords: Early China, cities, hereditary nobles, kinship, government

\title{
Las ciudades en el período pre-imperial
}

La aparición y difusión de las ciudades supuso una verdadera revolución en la organización social y política en el mundo antiguo. Las primeras formaciones proto-urbanas pueden verse en las aldeas del Neolítico que aparecen en la zona del río Amarillo aproximadamente 5000 años antes de nuestra era. El registro arqueológico da cuenta de la incipiente diferenciación de los palacios y centros de culto en estas aldeas (Lewis, 2006: 136). A lo largo de más de tres milenios, estas aldeas sirvieron de base para la consolidación de ciudades en el norte de China, pero la forma urbana más compleja hace su aparición recién a partir de la dinastía Shang (c. 1600-1045 a.e.c.), cuyos centros urbanos pasan a ser espacios donde se materializaba la concentración de poder político, religioso y económico.

Si bien estudios recientes muestran una génesis policéntrica de la cultura china, es cierto que las principales dinastías del período pre-imperial e imperial temprano, Shang, Zhou (1045-256 a.e.c.), Qin (221-209 a.e.c.) y Han (206 a.e.c.-220 e.c.) fundaron sus capitales y grandes ciudades en las llanuras del curso medio del río Amarillo, o bien sobre las márgenes del río Wei, uno de sus principales 
afluentes. Estas ciudades formaban parte de un entramado de gobierno fundado en lealtades de grupos de parentesco, cimentadas en rituales de carácter religioso que permitieron la expansión territorial de las dinastías pre-imperiales entre los siglos XIV y III a.e.c. Estas ciudades adquirieron relativa autonomía durante el período de Primaveras y Otoños (771-481 a.e.c.) de la dinastía Zhou, y fueron integradas a los nuevos estados territoriales del período de Estados Combatientes (481-221 a.e.c.). Tras la fundación del imperio a fines del siglo III, las ciudades cambiarían una vez más su función, transformándose en sedes administrativas a cargo de los magistrados designados por la corte central, por un lado, a la vez que fungían como centros de residencia de la aristocracia imperial.

Las primeras ciudades se presentan en China como centros de autoridad política. Tal como sugiere Trewartha, "la mayor parte de las ciudades de Shang probablemente fueron establecidas en conexión con alguna residencia oficial, y servía principalmente funciones políticas y militares” (Trewartha, 1952: 69). Una de las características del orden político de Shang es que su control territorial se sostenía a partir de la consolidación de una red de ciudades amuralladas, cuyo núcleo político eran los linajes locales ( $z u$ 族), vinculados con el soberano Shang por lazos familiares y/o religiosos (Chang, 1980). En el caso de China antigua e imperial, podemos observar que en gran medida las lógicas de parentesco se superponen con las de la estatalidad. Tal como lo señaló David Keightley "las prácticas de culto a los ancestros y la cultura política de Shang enfatizaban el linaje como fuente de autoridad. Dichas consideraciones basadas en el parentesco continuaron influenciando, si no saboteando, la operatoria de la administración burocrática a lo largo del período imperial" (Keightley, 1978: 214).

La corte Shang dependía más de los tributos de las ciudades aliadas y de las actividades de caza que de su capacidad para extraer impuestos de los campesinos con cierta regularidad. Es por eso que el rey visitaba estas ciudades con relativa frecuencia, lo cual permitía reafirmar las alianzas políticas que permitían sostener el control territorial. La capital, por lo tanto, no funcionaba como centro administrativo, ya que la centralidad política estaba encarnada en el cuerpo de los soberanos Shang, quienes podían pasar largos períodos fuera de su capital, ya sea en campañas militares, en excursiones de caza o en las visitas a las ciudades aliadas para ocuparse de los sacrificios a las deidades locales.

Cabe notar también que fuera del entramado de ciudades que se reconocían bajo la soberanía del rey de Shang estaban los cuadrantes externos (fang 方) habitados por poblaciones señaladas como culturalmente distintas y a menudo hostiles. En este sentido, Lewis advierte que el estado Shang no constituía una unidad territorial continua, sino más bien "una liga de pueblos aliados por sus vínculos familiares o por prácticas religiosas comunes, pueblos que existían en medio de asentamientos extranjeros y hostiles" (Lewis, 2006: 136).

Tras el declive de Shang a mediados del siglo XI a.e.c., uno de sus antiguos aliados lideraría la rebelión que dio lugar a la fundación de la dinastía Zhou. Los gobernantes de Zhou heredaron este entramado de ciudades aliadas que habían formado la ecúmene Shang, y lo expandió mediante la práctica de la 
investidura de nobles hereditarios (fengjian 封建), quienes pasaban a residir en una ciudad amurallada y tenían bajo su jurisdicción a una población determinada, sobre la cual podían extraer impuestos y demandar servicios militares o de trabajo estacionario.

En los primeros siglos de la dinastía Zhou se articula una primera distinción entre los nobles y demás habitantes de las ciudades, por un lado, y los campesinos que poblaban las aldeas vecinas, por el otro. Esta distinción entre el espacio rural, habitado por campesinos que debían pagar tributo en grano a su Señor, por un lado, y las ciudades, donde vivían los distintos grupos de la élite y donde se almacenaba la producción de los campos adyacentes, por el otro, va a mantenerse sin grandes cambios durante el extenso período imperial. Cabe notar también que la concentración de granos en las ciudades amuralladas permitía el mantenimiento de guarniciones militares, un elemento central en la consolidación de poder de la nobleza de Zhou. Según Trewartha, estas ciudades amuralladas constituyeron la estructura celular de la economía y de la sociedad en China y a partir de la multiplicación de estas unidades relativamente homogéneas se explica la expansión territorial de sus primeras dinastías (Trewartha, 1952: 70-71). Por su parte, Lewis sugiere que el orden Zhou dependía principalmente de la expansión militar, ya que la adhesión de los nobles dependía principalmente de la capacidad del soberano de garantizar el acceso a nuevos territorios como recompensa por los servicios prestados (Lewis, 2006: 138). La multiplicación de centros urbanos a lo largo de la llanura del río Amarillo y la expansión de la frontera agrícola en las zonas de la periferia resultó en la reconfiguración de la ecúmene Zhou.

Esto se debió en parte a que desde mediados del siglo VII a.e.c., las ciudades habían logrado incorporar a su espacio político a un número cada vez mayor de campesinos, tanto por los procesos de reclamación de tierras, como por las innovaciones administrativas que permitieron censar a la población circundante. También conviene notar el avance en las técnicas agrícolas y la mejora en las estructuras de regadío, que permitieron el incremento de la producción. Otras ciudades se formaron a partir de la necesidad de garantizar el cuidado y la protección de talleres especializados, o fuentes de recursos minerales.

Esta expansión territorial fue acompañada por una complejización del entramado administrativo proto-burocrático durante el período de Zhou Occidental (1045-771 a.e.c.). Los nobles de Zhou estaban vinculados a la corte central en un esquema jerárquico que definía su posición en el sistema de ciudades y poblados, a la vez que normaba sus rituales de corte y limitaba el tamaño de sus ejércitos, entre otras cosas. Los oficiales de la corte comenzaron a recibir territorios de igual manera, conformando así un segundo estrato de la nobleza. Este orden ideal se desintegraría a comienzos del siglo $V$ a.e.c., tal como señala Mencio en el capítulo “Wan zhang-xia 萬章下”:

Según lo establecido por el Hijo del Cielo, su territorio era de mil $l i$ cuadrados, mientras que los gong y hou recibían cien $l i$ cuadrados. A los bo les correspondían setenta $l i$ cuadrados, y a los zi y nan, cincuenta li cuadrados. Estas eran los cuatro órdenes de territorio. Aquellos (nobles) que no tenían siquiera cincuenta $l i$ cuadrados, no tenían derecho a entrevistarse con el Hijo del Cielo, sus territorios se incluían en los de uno 
de los nobles de mayor rango, y se le llamaba "territorio dependiente" (fuyong). Los grandes ministros (qing) del Hijo del Cielo recibían un territorio equivalente al de los hou, los grandes oficiales (daifu) recibían un territorio equivalente al de un bo, y los shi de primer nivel recibían territorios equivalentes a los de los zi y nan.

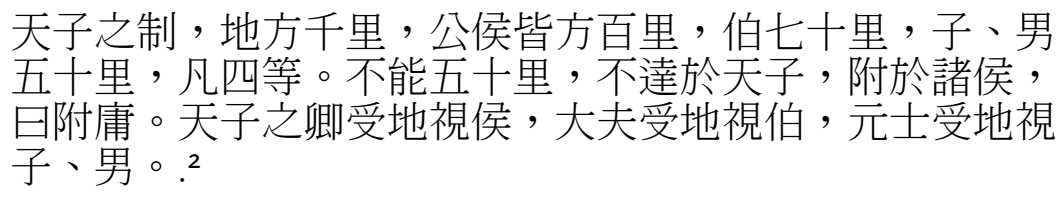

Con el declive de la corte de Zhou Occidental, en el período de Primaveras y Otoños (771-475 a.e.c.), los nobles hereditarios de las ciudades amuralladas se volvieron cada vez más independientes de la corte central, acumulando recursos económicos y militares, y principalmente, transgrediendo las normas simbólicas que definían la diferencia entre la corte central, y las cortes de los estados. Entre ellas, la más importante fue el uso del título de rey (wang 王) Asimismo, a partir de las prácticas de los cultos ancestrales, las capitales de los estados adquieren una nueva centralidad religiosa, que se expresaba en la persona del Señor y de su linaje en el Templo de los Antepasados, en los altares a las deidades de la tierra y los granos (sheji 社稷), término que a veces se utilizaba como equivalente con la unidad política, y de deidades que guardaban las murallas de la ciudad (Trewartha, 1952: 72). También ocurrió durante este período que los estados más poderosos de la periferia pasaron a incorporar un gran número de ciudades de menor tamaño a su espacio político, configurando nuevas fronteras.

Asimismo, podemos observar la creciente importancia de los habitantes de las ciudades en el esquema político de Zhou. Estos guoren 國人 aparecen en el registro histórico como un elemento fundamental en apoyo a los nobles de su localidad, sobre todo ante situaciones de crisis, tanto por amenazas externas como por disputas entre facciones de la élite local. En los distintos estados las familias aristocráticas y las ministeriales se enfrentaban para lograr tomar control de las instituciones de gobierno. La gran movilidad de los estratos inferiores de la aristocracia y la consolidación de un grupo de prestigio basado en el conocimiento, los shi 士, quienes pasaron a formar parte del cuerpo colegiado de consejeros y del entramado burocrático de las cortes locales, permitió una articulación de intereses con distintos grupos al interior de las ciudades. Las experiencias de grandes ministros como Guan Zhong 管仲 (725-645 a.e.c.) en el estado de Qi 齊, Zi Chan 子產 (siglo VI a.e.c.) en el estado de Zheng 鄭, o del mismo Confucio (551-479 a.e.c.) en el estado de Lu 魯 dan cuenta de las distintas dinámicas entre los aristócratas, los administradores del estado y los ciudadanos. Según Lewis, "si bien los ciudadanos en su conjunto nunca llegaron a tomar el control del gobierno, su apoyo era crucial para cualquiera que tuviera la intención de hacerlo" (Lewis, 2006: 145).

2 Mengzi, “Wan zhang-xia 萬章下”. Disponible en https://ctext.org/mengzi/wan-zhangii/ens\#n13553. 
Es importante señalar que la mayor parte de las ciudades de Zhou tenían una población relativamente pequeña en comparación con las ciudades del período imperial. Las principales ciudades de Zhou tenían algunas decenas de miles de habitantes. Quienes componían el núcleo de la actividad política en las ciudades de Zhou llegaban a tener trato directo entre distintas familias de ciudadanos, o al menos tenían referencias indirectas acerca de ellas. Las campañas militares, los mercados, las celebraciones en espacios públicos se transformaban en espacios de consolidación de una identidad asociada a la ciudad-estado. En particular, los rituales asociados con los altares de la tierra y los granos servían para renovar el compromiso entre los jefes de los principales linajes y el gobernante de la ciudad-estado (Lewis, 2006: 147). Los lugares públicos también servían de espacio de exhibición de los cuerpos, a menudo mutilados, de quienes habían transgredido la ley, convirtiéndose en una advertencia para quienes estuvieran pensando en rebelarse. ${ }^{3}$

Los convenios (meng 盟) entre los gobernantes y los jefes de las familias de ciudadanos comunes se hicieron más frecuentes en el registro del período de Estados Combatientes. Éste era un recurso para intentar asegurar su apoyo en un contexto de luchas intestinas en las ciudades entre los linajes nobles y ministeriales. Esta nueva relación, de tipo contractual, sirvió para incorporar al cuerpo político a grupos que no estaban vinculados por lazos de sangre (Lewis, 2006: 148). No obstante, como advierte Yuri Pines, no debemos caer en el error de asumir que estos habitantes de las ciudades, los guoren gozaban de los mismos derechos que sus contrapartes en las poleis de la Grecia clásica. Pines señala que en las ciudades del período de Primaveras y Otoños las asambleas eran un evento poco común, y se convocaban ante alguna necesidad particular, y no una institución política consolidada (Pines, 2009: 194-195). ${ }^{4}$

En China antigua se da un proceso particular, a la vez que se multiplican numerosos centros urbanos en el primer milenio antes de nuestra era, la población se mantuvo principalmente rural. Esta población rural no gozaba de las ventajas materiales y simbólicas asociadas a la vida en las ciudades, más bien su vida se parecía mucho a la de sus antecesores del Neolítico. Es por eso que las ciudades se concebían como espacios donde confluían la administración y el desarrollo de la cultura refinada, en contraposición a las comunidades rurales, que eran espacios prácticamente autogestados, donde los jefes de los principales linajes de cada aldea servían de intermediarios e interlocutores entre los magistrados de la jurisdicción y la población local.

Otro factor detrás del gran desarrollo urbano en la China pre-imperial fue la movilidad de las capitales en el período Zhou tardío. Esta mudanza de los

3 En un pasaje de las Analectas, uno de los discípulos de Confucio le sugiere que puede ejecutar a un adversario que había calumniado a Zi Lu, y exhibir su cadáver en el mercado o en la corte. Ver Yang Bojun 楊伯峻, 2009: 154-155.

4 Cabe notar que el mismo término se utiliza para describir los convenios sellados al interior de las ciudades-Estado y aquellos que los nobles de éstas sellaban para celebrar alianzas. Un estudio más completo de estos textos puede encontrarse en Crispin Williams (2014). 
centros de gobierno motorizaba las construcciones que replicaban las estructuras de residencia, gobierno y culto de las élites gobernantes en nuevos espacios urbanos. El ejemplo más claro de este fenómeno fue el establecimiento de la capital del estado de Qin en Xianyang a mediados del siglo IV a.e.c. No obstante, cabe notar que la antigua capital no era abandonada por completo, sino que a menudo conservaba un status privilegiado como "segunda" o "antigua" capital. Por último, cabe notar también que estas ciudades comenzaron a competir por atraer a los referentes de la cultura y el conocimiento oficialmente sancionado, artesanos, artistas, eruditos, expertos en lo religioso gravitaban hacia las cortes de los nobles.

A pesar de tratarse de un período marcado por los constantes conflictos militares, los estados de la desintegrada ecúmene Zhou experimentaron un notable crecimiento poblacional durante los siglos V y III a.e.c. Los hallazgos de las excavaciones arqueológicas realizadas en el norte de China a comienzos de la década de 1930 dan cuenta de que muchas de las principales ciudades del período de Estados Combatientes fueron el resultado de un proceso de expansión y construcción acumulativa a lo largo de los siglos precedentes. Algunas de las principales ciudades de Zhou fueron el resultado de la integración de dos o más ciudades de menor tamaño establecidas durante la dinastía Shang o en Zhou temprano, unidas a partir de la construcción de una muralla externa que las circundaba (guo 郭). A menudo el sitio de la ciudad principal pasaba a cumplir funciones palaciegas, manteniendo así su preeminencia simbólica al interior del nuevo espacio urbano, mientras que la otra parte de la ciudad quedaba relegada a la producción y al comercio, actividades consideradas de segundo grado.

Los nuevos espacios urbanos se caracterizaban por su relativa independencia de los códigos suntuarios que habían limitado las construcciones según el rango asociado a la ciudad. Es decir, durante el auge de Zhou, se suponía que, al menos idealmente, la extensión y la población de las ciudades y poblados de los nobles y oficiales debían ajustarse a su rango correspondiente. Por otra parte, mientras que en las ciudades del período de Primaveras y Otoños básicamente todos los hombres adultos participaban de los asuntos públicos, al menos ocasionalmente, el nuevo entramado urbano del período de Estados Combatientes implicaba una división espacial entre quienes participaban de los asuntos públicos, como la administración o los rituales, y quienes eran sujetos prácticamente pasivos del control por parte de los organismos de la administración de las cortes. Asimismo, los cambios en el gobierno de las ciudades marcan el reemplazo de los nobles vinculados a la casa real por administradores designados por la corte regional, y la resignificación del término shi que pasó de referirse al estrato más bajo de la clase aristocrática a designar al grupo de funcionarios al servicio de las cortes (Lewis, 2006: 152). En este sentido, podemos señalar que en los tres siglos finales de la dinastía Zhou los gobernantes de las principales ciudades-estados lograron aumentar su autonomía respecto a la corte central, a la vez que buscaron establecer mecanismos para controlar a las poblaciones que formaban parte de sus periferias.

Es también desde finales de Zhou que se fortalecen las murallas externas de las ciudades y se multiplican las defensas internas, a la vez que se establecen 
poblados y ciudadelas alrededor de las principales ciudades que servían de defensa. En el período de los Estados Combatientes nuevas formas arquitectónicas transformaron el espacio de las grandes ciudades. En este sentido, Wu Hung señala la aparición de estructuras como la plataforma (tai 台), la pagoda ( $g e$ 閣), la puerta de vigilancia (que 閲) y la torre de observación (guan 觀) (Wu, 1999: 653). En este período los desarrollos urbanos se caracterizan tanto por la inversión en estructuras que dan cuenta del prestigio y la riqueza de la élite gobernante, como por la preocupación por la defensa ante las múltiples amenazas. Es así que en el curso de los dos siglos finales de Zhou "la ciudad fue reformada y su estructura interna reconfigurada" (Wu, 1999: 651).

Muchas de estas nuevas ciudades sobrevivieron hasta la dinastía Han, e incluso algunas persisten hasta nuestros días. Sin embargo, su autonomía se vio seriamente mermada tras la conquista de Qin y la fundación de la primera dinastía imperial, siendo incorporadas a un nuevo esquema simbólico, ritual y administrativo. Lewis describe a este proceso como la "transformación del estado chino de una liga de ciudades-Estado bajo una dinastía teocrática a un gran imperio territorial [...] marcada por el cambio de la primacía ritual del templo ancestral a los cultos dedicados al Cielo y a las principales figuras del paisaje" (Lewis, 2006: 147).

\section{La centralidad de las primeras capitales imperiales: Xianyang y Chang'an}

Esta breve reseña de las instituciones de gobierno de las dinastías pre-imperiales es un trasfondo indispensable para enmarcar los cambios y continuidades en la composición demográfica y la organización territorial de las primeras dinastías imperiales. Éstas heredaron las ciudades y las redes de caminos que se habían desarrollado durante el período de Estados Combatientes, pero que, a partir de Qin, se integrarían en un nuevo esquema.

En lo que respecta al desarrollo urbano, un primer cambio fue que las primeras capitales imperiales fueron diseñadas y construidas con objetivos militares y políticos. Como indica Michèle Pirazzoli-t'Sertevens (2010: 173), ni la ciudad de Xianyang, capital de Qin, ni las capitales de Han, Chang' an 長安 y Luoyang 洛陽 fueron ciudades que se desarrollaron “naturalmente", es decir, a partir de la concentración de población por su acceso a recursos naturales o como puntos neurálgicos en vías comerciales, sino que fue la atracción que ejercieron una vez que se consolidaron como sedes del poder imperial lo que les permitió convertirse en grandes ciudades.

Lewis hace referencia a que esta nueva concepción de la capital imperial "se definía por su centralidad ritual, su estructura basada en los textos (clásicos), y su desconexión con cualquier tipo de vínculo regional o local” (Lewis, 2006: 135). Como capital del imperio, Xianyang fue diseñada como una representación del cosmos, y se erigieron edificios, templos, torres y plataformas que pretendían replicar el mapa celeste. Sin embargo, los altares del estado permanecieron en Yong y Longxi, las viejas capitales del estado de Qin. 
Los grandes estados del período de Estados Combatientes fueron puestos bajo el control directo de magistrados nombrados por la corte imperial. El primer emperador hizo grandes esfuerzos por eliminar los vestigios de la autonomía de las aristocracias tradicionales que subsistían en los estados. Una de las medidas en este sentido fue la relocalización forzada de la nobleza de los estados derrotados en la nueva capital imperial. Esto servía, por un lado, como forma de control político, mientras que, por otra parte, reflejaba la aspiración de la capital de ser el punto de encuentro y de superación de las identidades regionales. No es de extrañar que en el período imperial temprano se hayan articulado los discursos críticos de las costumbres ( $s u$ 俗) asociadas con las identidades regionales.

Por otra parte, se buscó subordinar a las antiguas capitales de los estados a un nuevo esquema de prefecturas y comandancias. En este sentido, una de las innovaciones del primer imperio burocrático fue que la jerarquía de las ciudades fue readecuada no a partir de su importancia histórica, demográfica o comercial, sino que ésta se empardaba con el nivel administrativo del magistrado que residía en ella. En la práctica, esto implicaba que las ciudades a las que se les había asignado un rango superior a menudo tenían menos población y riqueza que las capitales de comandancia (Trewartha, 1952: 73).

Pero aún con estas innovaciones administrativas, el primer emperador no podía confiar en que estas medidas garantizaran el orden recientemente establecido por lo que, durante su reinado, optó por recorrer personalmente el territorio, en lo que se conocía como los “tours de inspección” (xunshou 巡狩). A diferencia de la lógica que animaba a sus antecesores de la dinastía Shang en sus circuitos, el primer emperador no estaba preocupado por mantener la adhesión de una federación de linajes, sino más bien de demostrar su carácter divino y fundir su persona con la totalidad del territorio. De hecho, el circuito de inspección se articulaba con una serie de prácticas rituales auspiciadas por los clasicistas de la corte que inscribían a la figura imperial en la materialidad del paisaje (Kern, 2000). A pesar de estos esfuerzos, los estados mantuvieron una marcada identidad regional, que se hizo patente al momento de estallar las rebeliones que acabaron con la dinastía Qin.

Liu Bang se convirtió en el primer emperador de la dinastía Han en el 202 a.e.c. La ciudad de Chang' an 長安, cerca de la actual ciudad de Xi' an en la provincia de Shanxi (陝西) se convirtió en la capital durante Han Occidental. Chang'an fue reconstruida por los gobernantes de la nueva dinastía, quienes optaron por establecer su capital en la llanura central del río Wei, lejos de su localidad de origen en el estado de Chu, al este. De esta forma, el primer emperador y sus eruditos buscaban reforzar la idea de un centro imperial que articulaba un nuevo modelo cultural, uno que incorporaba algunas de las particularidades de los estados regionales, pero a la vez se distinguía de éstos por su carácter superador.

Pocas ciudades del mundo antiguo podían ostentar la población y la organización de Chang' an. Según estudios recientes, desde mediados de Han Occidental, el área metropolitana de Chang' an se estimaba en un millón de habitantes, mientras que sus áreas suburbanas sumaban otro millón. Esto representaba 
el 30\% de la población total, estimada en 60 millones de personas, pero probablemente la región concentraba hasta un $60 \%$ de la riqueza producida en el imperio (Nylan y Vankeerberghen, 2015: 18). La capital contaba además con un complejo de palacios donde residían el emperador, la familia imperial y los agentes del gobierno, funcionarios, y eunucos. En un relato preservado en los Registros del Historiador, uno de los principales asesores del fundador de Han, el erudito Xiao He 蕭何 (siglos III-II a.e.c.) le recomienda construir palacios para demostrar la autoridad imperial (Shiji 8, Lewis, 2006: 176).

Si bien quedan pocos rastros de su magnificencia original, se estima que las murallas externas hechas de tierra apisonada tenían una base de más de 100 metros de ancho, y se alzaban a más de 10 metros de alto. A esto debía sumarse un profundo foso que servía de protección a la capital imperial, aunque cabe notar que durante la mayor parte de la dinastía Han Occidental, las murallas dejaron de cumplir una función militar y pasaron simplemente a demarcar un área administrativa. Se estima que las áreas residenciales de las capitales de Han, así como los mercados estaban separados por muros, y cada área estaba supervisada por funcionarios de bajo rango o bien por jefes de familias notables. Ésta era la forma más conveniente y económica de garantizar el orden público en una ciudad tan grande (Lewis, 2006: 159).

Sin embargo, la capital de Han Occidental presentaba algunos problemas que fueron notados por observadores posteriores. El historiador Ban Gu, escribió un poema en el que critica las maravillas de la capital:

$\begin{array}{ll}\begin{array}{l}\text { Planificaron su fundamento para que dure cien } \\ \text { millones de años }\end{array} & \text { 圖皇基於億載, } \\ \begin{array}{l}\text { Imponente en su escala y majestuoso en su } \\ \text { construcción }\end{array} & \text { 度宏規而大起, } \\ \begin{array}{l}\text { Comenzó con el emperador Gao concluyó con el } \\ \text { emperador Ping, }\end{array} & \text { 肇自高而終平, } \\ \begin{array}{l}\text { Cada generación le sumó ornamentos y } \\ \text { aumentó su belleza }\end{array} & \text { 世增飾以崇麗, } \\ \begin{array}{l}\text { Extendiendo estos beneficios a lo largo de doce } \\ \text { reinados } \\ \begin{array}{l}\text { De modo que acabó en un exceso de lujos y } \\ \text { extravagancias }\end{array}\end{array} & \text { 歷十二之延祚, } \\ \end{array}$

La pacificación del territorio había hecho posible la extensión de las redes de comercio, y con eso la concentración de grandes fortunas en manos de familias de comerciantes. Durante la dinastía Han, el estado mantuvo una tensión constante respecto a estos grupos, a veces confiándoles las licencias de lucrativos monopolios, como el de la comercialización de la sal y los licores, a veces revocando estas licencias y persiguiendo a las familias acusándolas de evasión impositiva. Los principales debates respecto a la promoción o limitación de las 
actividades comerciales entre distintos grupos de consejeros de la corte imperial han quedado registrados en las célebres "discusiones sobre los monopolios de la sal y el hierro" (Yantielun 鹽鐵論). Es en este período que las familias poderosas y los comerciantes locales competían entre sí por demostrar su riqueza y su poder mediante la construcción de grandes complejos.

En este período se implementaron una serie de reglamentos que impedían a los comerciantes exitosos acceder a los signos visibles de status de los grupos de oficiales. Estas confrontaciones irían acompañadas de una intensa disputa textual sobre la legitimidad de las fortunas de los comerciantes, llevada adelante por eruditos de distintas escuelas. ${ }^{6}$ Sin embargo, cabe notar que los comerciantes que lograron congraciarse con la corte central llegaron incluso a obtener un reconocimiento simbólico en forma de títulos nobiliarios. Al habitar ciudades relativamente nuevas, que eran tanto sede administrativa como centros de la actividad comercial, y pudiendo codearse con la aristocracia y los funcionarios sin las restricciones que habían limitado su ascenso en Zhou, las familias de comerciantes del período imperial temprano tenían muchas más oportunidades de incorporarse a los estratos superiores de la élite imperial en pocas generaciones.

\section{Las redes de ciudades y los zhu hou wang de Han Occidental}

Fuera de la capital, el territorio de la dinastía Han fue dividido entre las comandancias y prefecturas heredadas del sistema de Qin, que eran administradas por funcionarios directamente designados por la corte central, por un lado, y una gran cantidad de territorios que fueron asignados a familiares del emperador, a quienes se les otorgaba el título de "príncipes" (wang 王), y otras localidades asignadas al cargo de nobles hereditarios (zhuhou 諸侯), por el otro. El territorio se dividió en unas doce provincias a cargo de gobernadores (shou 守 o taishou 太守), un número similar de “principados” (guo 國) y unas setenta localidades que fueron asignadas a nobles como recompensa a sus acciones meritorias $^{7}$ (Loewe, 2004: 308). Los príncipes y demás nobles hereditarios de la dinastía Han pasaron a residir en las capitales de los viejos estados o las principales ciudades de su jurisdicción.

Tras el establecimiento de la dinastía Han, su fundador Liu Bang, el emperador Gaozu, invistió con títulos nobiliarios a sus familiares y algunos de sus camaradas de armas más cercanos. Los nobles que fueron creados en este período gozaban de gran autonomía, contaban con un cuerpo de administradores, y

6 Ver las "Biografías de mercaderes" en Sima Qian 司馬遷, Registros del Historiador(Shiji 史記): $129.3253-3283$.

7 En nuestros escritos optamos por traducir el término wang 王como "príncipe" o "príncipes" cuando hace referencia a los familiares directos del emperador a quienes se les otorgaban territorios durante el período imperial. Para el período pre-imperial, traducimos wang como "rey", ya que hace referencia a la máxima autoridad de la dinastía gobernante. 
tenían la obligación de cobrar los impuestos correspondientes a su jurisdicción, remitiendo la mayor parte a la corte central y manteniendo un porcentaje para su propio tesoro. Sin embargo, en el transcurso de la primera década de su reinado, Gaozu se ocupó de eliminar a sus viejos aliados que habían conseguido mantener sus antiguos bastiones territoriales y reemplazarlos por familiares directos de la casa imperial. Sus sucesores continuaron esta tarea, de manera que para mediados del siglo II a.e.c. ya no quedaban territorios administrados por “príncipes de distinto apellido" ( yi xing wang 異姓王) ${ }^{8}$

Hay que tener en cuenta que, durante la dinastía Han, tanto los nobles de la casa Liu como los funcionarios designados por la corte central residían en las ciudades, lo que a menudo resultaba en tensiones entre quienes eran "carne y hueso” (gurou 骨肉) del emperador y sus magistrados. Por otra parte, la Rebelión de los Siete Principados a mediados del siglo II a.e.c. transformó parcialmente la lógica de las relaciones intra-familiares del clan de los Liu, ya que los príncipes del mismo apellido perdieron gran parte de sus territorios, y quedaron sujetos al control de los funcionarios de la corte central, quienes se ocupaban de reportar al emperador las transgresiones y los posibles intentos de sedición por parte de sus familiares más o menos cercanos.

La expansión territorial y la delicada situación del imperio tras la fundación de Han hizo necesario el control de las otras grandes ciudades que constituían bastiones en zonas agrícolas extremadamente fértiles y económicamente poderosas. Es por ello que, a lo largo del primer siglo de la dinastía, la corte central buscó garantizar el control de las “cinco capitales” $w u d u$ 五都. Éstas eran Luoyang, en la actual provincia de Henan, que más tarde fue la capital de Han Oriental; Handan, la capital del antiguo reino de Zhao, en la actual Hebei; Linzi, una de las ciudades más pobladas y prósperas del período de Zhou tardío, capital del antiguo reino de Qi en Shandong; la ciudad de Wan, en la actual provincia de Henan; y Chengdu, capital del antiguo reino de Shu en Sichuan.

Si bien todas estas ciudades se habían desarrollado durante el período preimperial, y a comienzos de Han eran igual de importantes que Chang'an, su función cambió por completo una vez que se consolidó el control territorial de la dinastía. La principal diferencia es que mientras que en el período de los Estados Combatientes estas ciudades estaban controladas por estados semiautónomos con escaso o nulo control de la corte Zhou, en el período imperial pasan a operar como sedes del gobierno provincial o local bajo el control de la corte central en Chang'an.

Aunque el registro arqueológico es limitado, existe una voluminosa producción textual que describe la opulencia de las ciudades en Han. Por ejemplo, se estima que la ciudad de Chengdu, la capital de Shu, en la región de la actual provincia de Sichuan, tenía una población de 76.000 hogares, aproximadamente 400.000 personas. El esplendor de esta gran ciudad quedó registrado en el " $f u$ de la

8 Corresponde señalar que, con una única excepción, el título de príncipes sólo fue otorgado a miembros de la casa imperial. Sin embargo, los demás títulos nobiliarios podían ser otorgados con mayor liberalidad para premiar a quienes hubiesen prestado servicios a la dinastía. 
capital de Shu” (Shu du fu 蜀都賦) del literato Zuo Si 左思 (250-305) de la dinastía Jin Occidental (266-420), que replicaba en gran medida la descripción poética de Chengdu atribuida al erudito Yang Xiong 揚雄 (53 a.n.e.-18 e.c.). Según el poema de Zuo Si, la ciudad contaba con más de 18 pórticos y unas 400 calles y sus mercados se destacaban por la disponibilidad de una gran variedad de productos de la región. ${ }^{9}$

También hubo voces críticas de las nuevas formas productivas y de sociabilidad en las grandes capitales de Han. Según el crítico Wang Fu 王符 (83-170), la multiplicación de grupos ociosos en la ciudad de Luoyang amenazaba la supervivencia del entramado social. En sus "Comentarios de un recluso" (Qian fu lun 潛夫論) escribe:

\footnotetext{
Si uno observa la situación actual en Luoyang, verá que aquellos que se ocupan de las tareas secundarias son diez veces más que los que labran la tierra, y que los vagos y buenos para nada son diez veces más que los que se dedican a tareas secundarias. Es así que, por cada hombre que labra la tierra, cien personas obtienen su alimento, por cada mujer que se dedica a la sericultura, cien personas obtienen su vestido, ¿cómo es posible mantenerlos a todos? En las cien prefecturas y las mil comandancias del reino, y también en sus miles de ciudades la situación es así.

今察洛陽, 浮末者什於農夫, 虛偽游手者什於浮末。是則 一夫耕, 百人食之, 一婦桑, 百人衣之, 以一奉百, 孰能 供之？天下百郡千縣，市邑萬數，類皆如此。10
}

Tras el interregno de Wang Mang a comienzos del siglo I e.c., la ciudad de Chang' an quedó en ruinas, por lo que los herederos de la casa de Liu decidieron mover su capital a Luoyang, inaugurando la dinastía Han Oriental o posterior.

\section{Reflexiones finales}

Las ciudades del período imperial temprano servían como centro administrativo de sus regiones correspondientes, pero estaban integradas en una jerarquía descendente desde la capital hasta los centros administrativos de menor escala. En cierto sentido, las demás ciudades, con sus nobles hereditarios y sus magistrados, podían considerarse réplicas en escala menor de la capital imperial. A diferencia del período de los Estados Combatientes, cuando las aristocracias de cada estado competían por construir palacios y templos cada vez más imponentes, las capitales de los principados volvieron a estar sujetas a limitaciones en cuanto al tamaño de sus palacios y edificios de gobierno, el número de oficiales de las cortes, y la capacidad de reclutar fuerzas militares. De esta manera se buscaba enfatizar la diferencia radical entre la capital imperial y las capitales de los principados, comandancias y demás jurisdicciones.

9 David Knechtges presenta una traducción completa y anotada de las "rapsodias de las tres capitales" de Zuo Si, entre las cuales se encuentra el "fu de la capital de Shu". Ver Knechtges, 1982: 341-372.

10 Disponible en https://ctext.org/library.pl?if=en\&res=2764. 
Michael Loewe advierte que, si bien los nobles creados durante las primeras décadas de la dinastía Han tenían la posibilidad de legar a sus descendientes masculinos tanto su título como la posibilidad de cobrar impuestos sobre las familias dentro del territorio que les había sido otorgado, "no deben ser considerados como miembros de una aristocracia hereditaria que se basaba enteramente o principalmente en el nacimiento; sino que más bien deben considerarse como un mecanismo administrativo para recompensar y retener las lealtades y controlar las rivalidades" (Loewe, 2004: 279). El extenso territorio de la dinastía Han pudo controlarse por cuatro siglos gracias a la participación de los príncipes y nobles hereditarios en el co-gobierno de sus territorios.

Como señala Griet Vankeerberghen (2013), el emperador podía asegurarse de contar con el apoyo de ambos grupos según sus necesidades, ya que los oficiales de la burocracia imperial garantizaban la administración regular, mientras que los príncipes de la familia imperial podían proveer recursos financieros o militares en contextos de crisis. De esta manera, las instituciones de los nobles hereditarios no sólo van a sobrevivir la caída de la dinastía Han, sino que van a ser uno de los pilares del sistema imperial burocrático por los dos milenios siguientes. 


\section{Q Bibliografía}

»Chang, K. C. (1980). Shang Civilization. New Haven-Londres: Yale University Press.

» Fan Ye 范嘩. (1981). Historia de Han posterior (Hou Han shu 後漢書). Taipei: Dingwen shuju.

» Keightley, D. N. (1978). The Religious Commitment: Shang Theology and the Genesis of Chinese Political Culture, en: History of Religions 17 (3): 211-225.

»Kern, M. (2000). The Stele Inscriptions of Ch'in Shih-huang: Text and Ritual in Early Chinese Imperial Representation. New Haven: American Oriental Society.

»Knechtges, D. R. (1982). Wen Xuan, or Selections of Refined Literature. Volume One: Rhapsodies on Metropolises and Capitals. Xiao Tong (501-531). Nueva Jersey: Princeton University Press.

»Lewis, M. E. (2006). The Construction of Space in Early China. Nueva York: State University of New York Press.

"Loewe, M. (2004). The Men Who Governed Han China. Companion to A Biographical Dictionary of the Qjn, Former Han and Xin Periods. Leiden: Brill.

» Nylan, M. y Vankeerberghen, G. (2015). Chang'an 26 BCE. An Augustan Age in China. Seattle: University of Washington Press.

"Pines, Y. (2009). Envisioning Eternal Empire: Chinese Political Thought of the Warring States Era. Honolulu: University of Hawai'i Press.

"Pirazzoli-t'Sertevens, M. (2010). Urbanism, en: Nylan, M. y Loewe, M. (eds.), China's Early Empires. A Re-appraisal. Cambridge: Cambridge University Press: 169-185.

»Sima Qian 司馬遷. (1981). Registros del Historiador (Shiji 史記). Taipei: Dingwen shuju.

» Trewartha, G. (1952). Chinese Cities: Origins and Functions, en: Annals of the Association of American Geographers 42 (1): 69-93.

»Vankeerberghen, G. (2013). Kinship and Kingship in Han China: The 120 CE Case against Liu Chang, King of Lecheng, en: Olberding, G. P. S. (ed.), Facing the Monarch: Modes of Advice in the Early Chinese Court. Cambridge, MA: Harvard University Asia Center: 203-236.

"Williams, C. (2014). Scribal Variation and the Meaning of the Houma and Wenxian Covenant Texts' Imprecation Ma yi fei shi 麻夷非是, en: Early China 37: 101-179.

"Wu, H. (1999). The Art and Architecture of the Warring States Period, en: Loewe, M. y Edward L. S., The Cambridge History of Ancient China from the Origins of Civilization to 221 BC. Cambridge: Cambridge University Press: 651-744.

»Yang Bojun 楊伯峻 (ed.) (2009). Analectas (Lunyu yizhu 論語譯注). Beijing: Zhonghua shuju. 
